\title{
Análise Fatorial Confirmatória da Prova de Avaliação de Competência Social Face à Carreira
}

\author{
Confirmatory Factor Analysis of the Perceived Social Competence Scale
}

\author{
Joana Carneiro Pinto ${ }^{*},{ }^{a}$, Maria do Céu Taveira ${ }^{b}$, Adelinda Candeias $^{c}$ \& Alexandra Araújo ${ }^{b}$ \\ ${ }^{a}$ Universidade Católica Portuguesa, Lisboa, Distrito de Lisboa, Portugal \\ ${ }^{b}$ Universidade do Minho, Minho, Distrito do Minho, Portugal \\ \& ' Universidade de Évora, Évora, Distrito de Évora, Portugal
}

\begin{abstract}
Resumo
A competência social diz respeito à forma como as pessoas percebem e atuam face a uma variedade de situações sociais. Este estudo avalia as características métricas da Prova de Avaliação de Competência Social face à Carreira (PACS-Car). Participaram 572 adolescentes $(M=13,28$ anos; $D P=0,59)$, do $8^{\circ}$ ano de escolaridade ${ }^{1}$ de escolas portuguesas. Os resultados da Análise Fatorial Confirmatória apontam para uma solução fatorial constituída por oito fatores. Os índices globais de adequação registados para o modelo são bastante robustos, comprovando a sua qualidade no ajustamento aos dados empíricos $\left(X^{2} / g l=4,097, \mathrm{CFI}=0,895, \mathrm{GFI}=0,851, \mathrm{RMSEA}=0,079\right)$. Estes resultados estimulam ao desenvolvimento de pesquisa e intervenções que abordem as competências sociais para a promoção de comportamentos socialmente inteligentes de adaptação, progresso, e sucesso de carreira dos jovens. Palavras-chave: Habilidades sociais, orientação vocacional, adolescentes, análise fatorial confirmatória.
\end{abstract}

\begin{abstract}
Social competence is concerned to how people perceive and act in a variety of social situations. This study evaluates the psychometric characteristics of the Perceived Social Competence Scale (PSC-Car). Participants were 572 Portuguese adolescents $(M=13.28$ years; $S D=.59)$ who are in the $8^{\text {th }}$ grade. Results of the Confirmatory Factor Analysis suggest a factorial solution consisting of eight factors. The global indices of fitness of the model are quite robust, proving that it is a good solution in the adjustment of empirical data $\left(X^{2} / d f=4.097, \mathrm{CFI}=.895, \mathrm{GFI}=.851, \mathrm{RMSEA}=.079\right)$. The results stimulate the development of new researches and interventions that approach social skills in order to develop socially intelligent behaviors for career adaptation, advancement and success of young people. Keywords: Social skills, vocational guidance, adolescents, confirmatory factor analysis.
\end{abstract}

A competência social pode ser definida como a capacidade para selecionar e implementar os recursos cognitivo-comportamentais considerados mais adequados para lidar com os desafios impostos por uma situação interpessoal específica (Candeias, 2008). Um comportamento socialmente inteligente envolve processos psicológicos e contextuais, como: (a) processos motivacionais, que incluem interesse e preocupação em realizar objetivos (Ford, 1986), as crenças pessoais relativas às próprias capacidades (Bandura, 1986), e a expressividade emocional, ou seja, as reações aos sucessos e/ou fracassos sociais constituem um importante fator para desencadear ou não esforços para se ser socialmente inteligente (Ford, 1995); (b) processos relacionados com as capacidades (skill-related processes), que incluem o conhecimento social

" Endereço para correspondência: Centro de Investigação em Psicologia, Universidade do Minho, Campus de Gualtar, Braga, Portugal 4710-057. E-mail: joanacpinto_@ hotmail.com

${ }^{1} \mathrm{O} 8^{\circ}$ ano de escolaridade no ensino português corresponde à $7^{\mathrm{a}}$ série do ensino fundamental, no sistema de ensino brasileiro. sobre comportamentos, a capacidade de codificação de informação social, a capacidade de planeamento e de resolução de problemas sociais enquanto preditores do comportamento social eficaz (Ford, 1982); e (c) processos contextuais, que integram a congruência entre o contexto social, os objetivos e as capacidades pessoais, incluindo ainda os recursos informativos e materiais e um clima emocional positivo (Ford, 1995).

Por recursos cognitivo-comportamentais entendem-se os comportamentos e as atitudes, por exemplo, de assertividade, autocontrolo, empatia, responsabilidade, e cooperação (Gresham \& Elliot, 1990), aprendidas e desenvolvidas ao longo da vida, em diferentes contextos sociais, desde a família, passando pelo grupo de pares, até ao ambiente profissional (Del Prette \& Del Prette, 2005; Fumo, 2009; Murakami, Murray, Sims, \& Chedzey, 2009). Para a implementação destes recursos contribuem ainda as informações relativas ao/s outro/s: as suas emoções, interesses, pensamentos e comportamentos, bem como, as interações entre estes aspetos e o próprio indivíduo (Candeias \& Almeida, 2005). Estes recursos são selecionados 
com base na avaliação que a pessoa faz da especificidade da situação, e postos em ação para resolver problemas interpessoais concretos, contribuindo para o bem-estar pessoal e social (Tyler, 1984).

A competência social favorece uma adaptação positiva dos indivíduos aos contextos, contribuindo para a realização e sucesso em diferentes domínios da vida (e.g., Carvalho, 2011; Cecconello \& Koller, 2000; Gresham \& Elliot, 1984; Pinto, Taveira, Candeias, \& Araújo, 2011; Sternberg \& Grigorenko, 2003). Por exemplo, a nível académico, diferentes estudos com crianças e jovens têm procurado desvelar a relação entre um repertório cognitivo-comportamental bem desenvolvido, e a adaptação à escola, o sucesso e a realização académicas, e o desenvolvimento de relações interpessoais positivas com professores e pares (e.g., Alexander, Entwisle, \& Dauber, 1993; Bandeira, Del Prette, Del Prette, \& Magalhães, 2009; Fumo, 2009; Henricsson \& Rydell, 2006; Ladd, 1999; Welsh, Parke, Widaman, \& O’Neil, 2001). De um modo geral, os resultados da pesquisa sobre o tema indicam que as competências sociais atuam como um fator favorável à adaptação académica (Alexander et al., 1993). Apesar de resultados contraditórios nos diferentes estudos, o comportamento socialmente competente parece influenciar, e ser influenciado, também, pelo nível de desempenho académico (Bandeira et al., 2009; Fumo, 2009; Malecki \& Elliot, 2002; Molina \& Del Prette, 2006). Além disso, verifica-se que os alunos com comportamentos socialmente competentes desenvolvem relações interpessoais mais positivas com os seus professores e pares (Henricsson \& Rydell, 2006), sendo alvo de maior aceitação, e por este motivo, menos suscetíveis de experienciar isolamento ou rejeição social (Henricsson \& Rydell, 2006; Ladd, 1999).

A revisão da literatura neste domínio permite identificar três tendências na investigação. Por um lado, verifica-se um foco na identificação das principais características e delimitação do conceito de competência social, por oposição a outros conceitos análogos (e.g., competência quotidiana, inteligência social, personalidade, desempenho social, e habilidades sociais; Del Prette \& Del Prette, 2005; Greenspan \& Driscoll, 1997). A este respeito, os diferentes estudos têm demonstrado que o construto da competência social é multidimensional e dinâmico, sendo constituído por uma diversidade de componentes pessoais (e.g., cognitivos e emocionais, como a perceção de sucesso e fracasso), e contextuais (e.g., recursos e obstáculos percebidos na própria situação social), que interagem entre si, influenciando-se mutuamente (Candeias \& Almeida, 2005; Lemos \& Meneses, 2002; Sternberg \& Grigorenko, 2003).

Por outro lado, constata-se um interesse em torno do desenvolvimento, validação, e estudo sistemático de instrumentos destinados à avaliação das atitudes e comportamentos que integram o construto. A este nível, os diversos autores parecem ser unânimes quanto à necessidade de esta avaliação ser multimodal (Feitosa, 2007; Fumo, 2009;
Lemos \& Meneses, 2002). Isto é, realizada a partir de múltiplos informadores (e.g., professores, pais, e pares), indicadores (e.g., capacidade de iniciativa, confiança em si e nos outros, autoestima), e métodos (e.g., provas, escalas, entrevistas, inventários, e observação direta). Algumas das escalas e provas existentes neste domínio têm vindo a ser alvo de investigação sistemática, em contextos culturalmente distintos, e a partir de versões de auto e heteroavaliação (e.g., Bruder \& Chen, 2007; Candeias \& Almeida, 2005; Gouley, Brotman, Huang, \& Shrout, 2008; Lemos $\&$ Meneses, 2002; Schoon, 2009).

Finalmente, a terceira grande tendência caracteriza-se pelo foco na exploração da relação entre a competência social e outras variáveis. A este respeito, além da já referida ligação entre a competência social e variáveis do meio académico (e.g., Welsh et al., 2001), salienta-se a relação deste construto, por exemplo, com a qualidade de vida (Carneiro, Falcone, Clark, Del Prette, \& Del Prette, 2007), a capacidade de resiliência e adaptação (Cecconello \& Koller, 2000), ou o bem-estar psicológico (Segrin, Hazel, Donnerstein, Taylor, \& Domschke, 2007). Em alguns casos, estas variáveis são analisadas tendo em consideração grupos populacionais e/ou problemáticas específicas, como é o caso de crianças com perturbações de desenvolvimento (e.g., autismo, deficiência mental; Cotugno, 2009; Gresham, Sugai, \& Horner, 2001), ou de idosos institucionalizados (Carneiro et al., 2007).

De salientar a escassez de estudos que abordem o papel das competências sociais no desenvolvimento vocacional, não obstante a sua importância para compreender os processos de adaptação, desenvolvimento, e sucesso de carreira (e.g., Bandura, Barbaranelli, Caprara, \& Pastorelli, 2001; Fagoulis \& Phillips, 2011; Murakami et al., 2009; Pinto et al., 2011; Salmela-Aro, Tolvanen, \& Nurmi, 2011). Por exemplo, no estudo desenvolvido por Bandura e colaboradores (2001), com 272 alunos entre os 11 e os 15 anos de idade, foi analisada a relação entre as influências sociocognitivas (crenças de autoeficácia social) e as aspirações e trajetórias de carreira. Os resultados obtidos indicam que, apesar da perceção dos jovens acerca da sua capacidade para desenvolver comportamentos socialmente competentes não determinar, diretamente, as suas decisões sobre as trajetórias ocupacionais futuras, influencia-as indiretamente, por meio das aspirações. Por seu turno, os estudos desenvolvidos por Murakami e colaboradores (2009) e Salmela-Aro e colaboradores (2011), com jovens universitários demonstram, por um lado, que apesar das competências técnicas e científicas serem fundamentais para o exercício adequado de uma profissão, a generalidade dos jovens carece de um treino ao nível das competências sociais, o que traz dificuldades acrescidas na transição escola-trabalho. Por outro lado, o investimento das universidades no apoio ao desenvolvimento de estratégias sociais adequadas pode atuar como um fator protetor na adaptação destes jovens à carreira, contribuindo para níveis mais elevados de envolvimento no trabalho e níveis mais reduzidos de burnout. 
Pinto, J. C., Taveira, M. C., Candeias, A. \& Araújo, A. (2013). Análise Fatorial Confirmatória da Prova de Avaliação de Competência Social Face à Carreira.

Neste domínio, um dos principais desafios que o século XXI coloca aos serviços de carreira é contribuir, em qualquer fase do ciclo de vida de uma pessoa, para o desenvolvimento de projetos de vida pessoalmente significativos, mas também, eticamente refletidos, com vista a constituírem projetos socialmente sustentáveis e promotores do bem comum (e.g., Duarte et al., 2009; Guichard, 2011; Savickas et al., 2009). É, de facto, na interação entre a pessoa e a sociedade que ocorre o desenvolvimento humano, nas suas mais diversas dimensões (e.g., pessoal, vocacional, social). Assim, a construção que cada pessoa faz de si, e dos seus projetos de vida, resulta das interações com os diferentes atores dos contextos em que se encontra inserido (Duarte et al., 2009; Guichard \& Huteau, 2001; Savickas, 2005; Taveira et al., 2010). Por este motivo, a intervenção desenvolvida neste âmbito deve contemplar uma abordagem às competências sociais, enquanto condição pessoal que favorece um questionamento ético aquando da construção e desenvolvimento de projetos de vida (Taveira et al., 2010). Esta nova abordagem apoia a reconceptualização dos objetivos individuais de carreira, adicionando, ao foco intrapessoal, um foco interpessoal. Por outras palavras, para além da necessidade de obtenção de realização e satisfação pessoal, o indivíduo é convidado a refletir sobre o impacto interpessoal e social dos seus projetos e realizações (e.g., impacto na família, amigos e companheiros, nas instituições, na sociedade mais geral).

Este estudo insere-se nesta perspetiva e visa contribuir para colmatar a ausência de investigação sobre o papel da competência social percebida no questionamento ético dos projetos de vida. Para o efeito, foi desenvolvida uma medida de avaliação da competência social face à carreira (PACS-Car; Araújo, Taveira, \& Candeias, 2009; Candeias, 2001), destinada a jovens entre os 12 e os 18 anos, cujas características métricas são apresentadas e discutidas em seguida.

\section{Método}

\section{Participantes}

Os participantes deste estudo são 572 adolescentes que se encontram a frequentar o $8^{\circ}$ ano de escolaridade ${ }^{2}$, em seis estabelecimentos de ensino Básico e Secundário, com representação das zonas norte (Viana do Castelo, $n=172$; e, Braga, $n=231$ ), centro (Aveiro; $n=92$ ), e sul de Portugal (Lisboa, $n=104$; e, Évora $n=73$ ). Destes adolescentes ( $n=568), 295$ são raparigas e 273 são rapazes, com uma média de idades de 13.28 anos $(D P=0,59$; Min=11; $\operatorname{Max}=16)$.

\section{Instrumento}

A Prova de Avaliação de Competência Social face à Carreira (PACS-Car; Araújo et al., 2009) resulta da

\footnotetext{
${ }^{2}$ Ver nota anterior.
}

adaptação da Prova de Avaliação de Competência Social (PACS; Candeias, 2001) a situações de carreira.

Originalmente, a prova foi construída com o objetivo que cada situação proporcionasse referências comportamentais e contextuais específicas e detalhadas, podendo recorrer a perceções de pares, de supervisores (professores, chefes, entre outros), e do próprio, a respeito da competência social em situação específica, manifestada pelo indivíduo. O instrumento original é composto por seis situações sociais hipotéticas que requerem uma grande variedade de skills comportamentais, emocionais e cognitivas. Para o desenvolvimento desta prova tivemos como referência o Social Competence Nomination Form (SCNF; Ford, 1982), considerado um dos testes de avaliação mais fiáveis e válidos no âmbito das competências sociais. Tivemos ainda em conta os contributos de outros autores, como Barnes e Sternberg (1989) que alteraram o formato inicial do SCNF, introduzindo novas questões com o objetivo de recolher o índice de complexidade percebido pelo indivíduo na resolução de cada situação, oscilando desde muito complexo até ao nada complexo (Candeias, 2008). Em termos gerais, a PACS, na versão original, avalia competência social em situações interpessoais com pares e adultos significativos, em situações estruturadas e formais e em situações íntimas e informais. Todas estas características foram respeitadas na versão da Prova de Avaliação de Competência Social face à Carreira (PACS-Car). Para o efeito teve-se em conta, ainda, a natureza das atividades de Educação para a Carreira que devem expressar

um esforço do Sistema Educativo e de toda a comunidade dirigido a contribuir para a reforma educativa, ajudando as pessoas com atividades de infusão curricular, a relacionar a educação e o trabalho e a adquirir competências gerais para um desenvolvimento da carreira positivo, de forma a permitir a cada um fazer do trabalho remunerado ou não, uma parte significativa do seu estilo de vida (Hoyt \& Shylo, 1987, p. 34).

$\mathrm{O}$ processo de adaptação iniciou-se pela construção de 12 situações sociais novas, que refletissem, por um lado, a natureza das atividades de educação para a carreira ajustadas ao grupo etário ao qual se destina aprova, e ao mesmo tempo, respeitassem as características da PACS. A construção destas novas situações foi elaborada por uma doutorada em Psicologia Vocacional, investigadora da equipa, e especializada em temas de desenvolvimento vocacional na infância e educação para a carreira, após um estudo da PACS. Em seguida, as doze situações foram enviadas à autora da PACS e a outra investigadora para identificação e reconhecimento do tipo de situação, e apreciação do conteúdo, quer em termos da avaliação das competências sociais, quer da natureza da atividade proposta de educação para a carreira. Com base nesta avaliação foram escolhidas seis situações, que se ajustavam aos dois requisitos e obtiveram acordo superior a $50 \%$ entre juízes. Estas foram enviadas novamente às duas juízas agora já com a equivalência à respetiva situação original, que avaliaram de novo a sua adequação conceptual e a fa- 
cilidade/dificuldade da mesma para a faixa etária em jogo, revendo a sua formulação, no sentido do aperfeiçoamento. A prova foi então administrada a seis jovens, de ambos os sexos e com idades compreendidas entre os 12 e 16 anos, para avaliação de aspetos, tais como, a sua compreensão e tempo de administração.

A PACS-Car pretende avaliar o grau de desempenho/ competência e o grau de facilidade/dificuldade percebidos para lidar com situações interpessoais distintas que envolvem pares e adultos significativos, com um caráter mais formal ou informal, no âmbito da carreira. A Tabela 1 apresenta a situação exemplo e as 6 situações sociais adaptadas ao contexto de carreira: (a) situação de trabalho de casa, (b) situação de duplo encontro, (c) situação de nomeação para liderar um grupo, (d) situação de um(a) amigo(a) conselheiro(a), (e) situação de reunião de pais, e, (f) situação de um estudante visitante.
Para cada situação social são colocadas quatro questões: (a) esta situação para mim seria difícil, (b) esta situação para mim seria fácil, (c) o meu desempenho nesta situação seria pobre, e, (d) o meu desempenho nesta situação seria excelente. A resposta a cada questão é dada através de uma escala de tipo Likert, de cinco pontos, em que 1 representa "nunca" e 5 representa "sempre". Os itens relativos à autoavaliação de dificuldade e desempenho pobre na tarefa têm necessidade de ser invertidos previamente à realização de qualquer análise estatística.

Estudos prévios desenvolvidos com a medida original PACS (Candeias, 2008; Candeias \& Almeida, 2005) apontam para uma solução fatorial de seis fatores, correspondentes às seis situações sociais em contexto académico referidas. Estas explicam $64 \%$ da variância total do instrumento, com cargas fatoriais elevadas que variam entre 0,46 e 0,85 . A consistência interna das subescalas é bastante adequada, com alphas de Cronbach superiores a 0,75 , em todas as medidas.

Tabela 1

Prova de Avaliação de Competência Social em Contexto de Carreira

Situação exemplo

Vai haver um concurso de apresentação de trabalhos na sua escola. Esta turma está a preparar um trabalho para apresentar oralmente no dia do concurso. Cada turma deve escolher um porta-voz que apresente e defenda o trabalho em público.

1. Situação de trabalho de casa - itens 1.1 a 1.4

O psicólogo/psicóloga da escola marcou encontros semanais com os(as) alunos(as) para a realização de actividades de orientação e desenvolvimento vocacional. Nestes encontros, os alunos têm reflectido acerca de si próprios(as), sobre aquilo que sabem e necessitam de saber acerca do mundo escolar e das profissões, e ainda acerca do que precisarão para tomar decisões sobre o seu futuro enquanto estudantes e trabalhadores. A tarefa desta semana é individual e consiste na realização de uma descrição de si próprio(a), onde os alunos deverão salientar o que mais gostam, o que sabem fazer melhor, o que mais valorizam, e quais os seus objectivos, para o futuro. Os(as) alunos(as) demonstram dúvidas acerca desta tarefa e pedem ao(à) psicólogo(a) para serem ouvidos. O(a) psicólogo(a) concordou em conversar com um representante dos alunos, acerca do assunto e das dúvidas levantadas.

2. Situação de duplo-encontro - itens 2.1 a 2.4

O irmão do teu(tua) melhor amigo(a) está numa fase menos boa, porque não tem muita vontade de ir às aulas, estudar e comportar-se correctamente com os professores e colegas. Tem demonstrado dificuldade em perceber a utilidade da escola e dos diplomas escolares. Como bom amigo/boa amiga que és, o(a) teu(tua) amigo(a) pede a tua ajuda para tentar animar o seu irmão, e ajudá-lo a perceber porque é que a escola é importante para o futuro, quer enquanto pessoas, quer enquanto trabalhadores. Assim, o teu amigo pede que o acompanhes a um encontro com o seu irmão, combinando que a dada altura se retira, e te deixa a sós com o seu irmão, para poderem conversar.

3. Situação de nomeação para liderar um grupo - itens 3.1 a 3.4

A direcção da escola decidiu criar uma hora semanal obrigatória em que os alunos devem participar num clube. Têm à escolha várias alternativas, desde clubes desportivos, de música, de jornalismo, matemática, inglês, artes plásticas, de experiências nas ciências naturais e físico-químicas, ou mesmo de ajuda social na comunidade. Cada director de turma pediu que fosse eleito um líder ou representante da turma, para participar na comissão de formação dos clubes e ajudar os colegas a tomarem uma boa decisão e a escolherem o clube mais adequado a cada um(a). Este aluno(a) deve estar preparado(a) para ajudar os colegas a tomar a decisão, e explicar aos colegas como se tomam boas e más decisões na escola.

4. Situação de um(a) amigo(a) conselheiro(a) - itens 4.1 a 4.4

Alguns amigos(as) estão praticamente decididos(as) acerca do curso superior a frequentar, após o $12^{\circ}$ ano, mas procuram um amigo(a) para os ajudar a planear a melhor maneira de ter sucesso na sua escolha. Esse amigo(a) deverá ser capaz de escutar e de dar conselhos acerca do que deverá ser feito para alcançar bons resultados nessa decisão. 
Pinto, J. C., Taveira, M. C., Candeias, A. \& Araújo, A. (2013). Análise Fatorial Confirmatória da Prova de Avaliação de Competência Social Face à Carreira.

5. Situação de reunião de pais - itens 5.1 a 5.4

O professor de Língua Portuguesa pediu aos alunos que elaborassem um trabalho acerca das profissões dos seus pais. Neste trabalho, os alunos deverão descrever o que já conhecem acerca destas profissões: podem ser incluídas entrevistas, fotografias, ou artigos e imagens retiradas da Internet. Depois do trabalho elaborado, no final do período, será convocada uma reunião de pais, onde os alunos terão a oportunidade de apresentar, quer aos professores, quer aos seus pais, o resultado do seu trabalho. De modo a melhor organizar este evento, a turma decidiu escolher um representante de cada grupo, que fizesse uma introdução aos pais, do trabalho realizado pelo respectivo grupo.

6. Situação de um estudante visitante - itens 6.1 a 6.4

Os alunos desta escola estão muito entusiasmados com a realização de uma feira acerca do mundo profissional. Ao longo dos três dias reservados para esta feira, os alunos poderão assistir a apresentações de profissões por vários trabalhadores convidados. Cada director de turma pediu à sua classe que escolhesse um representante. Esse aluno(a) deverá ser um porta-voz da turma, colocando perguntas aos profissionais, acerca do seu trabalho no dia-a-dia.

\section{Procedimento}

Os dados foram recolhidos no início do segundo período escolar do ano letivo de 2010/2011. Após um contacto inicial com as Direções das escolas selecionadas para o estudo, foram conduzidas reuniões com as mesmas, para apresentação do projeto de investigação. Este foi apresentado também aos pais, professores e psicólogos dos alunos. Após a obtenção dos consentimentos informados dos encarregados de educação e dos alunos, estes últimos preencheram a PACS-Car em contexto de sala de aula, ao longo de cerca de 20 minutos, na presença do professor de uma disciplina e de uma psicóloga, investigadora do projeto. Foi assegurada a confidencialidade e o recurso a especialistas no tratamento dos dados.

\section{Análises}

Os dados recolhidos através do preenchimento da PACS-Car foram tratados com o software estatístico PASW Statistics, versão 18.0. Procedeu-se à análise da distribuição dos itens e da consistência interna das subescalas, e à análise fatorial exploratória de todos os seus itens. Foi utilizado o software AMOS (v. 19) para realizar a Análise Fatorial Confirmatória. Foram analisados os coeficientes de assimetria e curtose univariada e multivariada, e a existência de outliers, através da distância quadrada de Mahalanobis. A qualidade dos modelos fatoriais teve em consideração os seguintes índices e respetivos valores de referência (Marôco, 2010): $X^{2} / g l$, CFI (Comparative Fit Index; >0,90), GFI (Goodenss-of-Fit-Index; >0,90), RMSEA (Root Mean Square Error of Approximation; <0,08), $P[\mathrm{rmsea} \leq 0,05]$. Nos testes utilizados, foram considerados estatisticamente significativos os resultados com um valor de significância inferior a $0,05(p<0,05)$.

\section{Resultados}

A análise descritiva dos itens da PACS-Car indica a existência de uma distribuição das respostas dos participantes por todas as possibilidades da escala de resposta. Contudo, os valores médios obtidos em cada item afastam-se significativamente do ponto médio teórico. A interpretação das notas $z$ associadas aos coeficientes de assimetria e curtose sugere uma distribuição assimétrica negativa e platicúrtica face à generalidade dos itens. A análise da validade interna da escala indica que o poder discriminativo dos itens supera o limiar crítico de 0,20 , variando entre 0,43 e 0,59 , em todos os itens. A análise da consistência interna dos itens indica a inexistência de problemas de homogeneidade, com coeficientes alpha de Cronbach superiores ao limiar crítico de $0,70 \mathrm{em}$ todos os itens, oscilando entre 0,89 e 0,90 .

$\mathrm{Na}$ análise da estrutura fatorial da escala foi escolhida uma solução oblíqua e sem especificação de fatores, por ser a mais clara e congruente com o modelo conceptual original da PACS. Os testes da matriz de intercorrelação, como o teste de Keyser-Meyer-Olkin (KMO $=0,803)$, e o teste Bartlett $\left(X^{2}=8509,503 ; g l=276 ; p=0,001\right)$, apresentam valores adequados. A estrutura fatorial exploratória da PACS-Car é constituída por oito fatores com valor-próprio igual ou superior à unidade, explicando no seu conjunto $80,45 \%$ da variância dos itens. Seis dos oito fatores remetem para uma situação social específica. O fator 1 é constituído pelos itens da situação de reunião de pais (itens 5.1 a 5.4); o fator 3 é constituído pelos itens da situação de amigo(a) conselheiro(a) (itens 4.1 a 4.4); o fator 4 é composto pelos itens da situação de nomeação para liderar um grupo (itens 3.1 a 3.4); o fator 5 é composto pelos itens da situação de duplo encontro (itens 2.1 a 2.4); o fator 6 engloba os itens da situação de trabalho de casa (itens 1.1 a 1.4); e, o fator 8 engloba os itens da situação de um estudante visitante (itens 6.1 a 6.4). Por seu lado, os fatores 2 e 7 incluem, respetivamente, todos os itens de desempenho pobre (itens 1.3, 2.3, 3.3, 4.3, 5.3, e 6.3), e todos os itens de desempenho excelente (itens 1.4, 2.4, $3.4,4.4,5.4$, e 6.4 ), através de todas as situações sociais. Os fatores 4 e 8 são os únicos cujos itens apresentam uma saturação negativa. Relativamente à consistência interna da estrutura fatorial escolhida, foram registados resultados superiores ao limiar crítico de 0,70 (Nunnally, 1978) em todas as subescalas, variando entre 0,80 no fator 6 e 0,84 no fator 1 , o que comprova a ausência de problemas de homogeneidade entre as respostas dos participantes aos itens e o construto avaliado na prova.

A Análise Fatorial Confirmatória (AFC) realizada teve como principal finalidade testar o grau de ajustamento global do modelo teórico subjacente à PACS-Car aos 
dados empíricos recolhidos. Foram testados dois modelos principais, e respetivos modelos alternativos. A Tabela 2 apresenta uma síntese dos índices de adequação para o modelo de medida original da PACS, assim como, para o modelo de desempenho social na carreira (PACS-Car), resultante do estudo fatorial exploratório, e ainda, os modelos alternativos formulados com base nas sugestões de modificação decorrentes das AFC.

\section{Modelo Geral - Seis Fatores}

Foi testado o modelo de seis fatores, correspondentes às seis situações sociais da prova subjacente à PACS, e dois modelos alternativos: 1.1 Modelo geral com correlação entre todos os fatores; e, 1.2 Modelo geral com correlação entre todos os fatores e sem outliers.

A Tabela 2 apresenta uma síntese dos índices de adequação registados. Verifica-se que modelo geral apresenta índices de adequação insatisfatórios $\left(X^{2}\right)$ $g l=15,455$, CFI $=0,565$, GFI $=0,506$, RMSEA $=0,163$, $P[$ rmsea $\leq 0,05]<0,001)$. Na sequência desta análise, e por terem sido detetados casos extremos multivariados, através da distância quadrada de Mahalanobis, foram desenvolvidos dois novos modelos alternativos, com correlação entre todos os fatores, com (modelo 1.1) e sem outliers (modelo 1.2). Este último modelo apresenta os melhores resultados $\left(X^{2} / g l=8,914, \mathrm{CFI}=0,716, \mathrm{GFI}=0,649, \mathrm{RMSEA}=0,127\right.$, $P[$ rmsea $\leq 0,05]<0,001)$, apesar de não aceitáveis.
Modelo de Desempenho Social na Carreira-Oito Fatores

Seguidamente, foi testado o modelo de oito fatores seis situações sociais, um fator de desempenho excelente e um fator de desempenho pobre -, derivados da AFE, e um conjunto de três modelos alternativos: 2.1 Modelo de desempenho social na carreira com correlação entre o desempenho pobre e o desempenho excelente; 2.2 Modelo de desempenho social na carreira com correlação entre todos os fatores; 2.3 Modelo de desempenho social na carreira com correlação entre todos os fatores e sem outliers.

$\mathrm{O}$ modelo de desempenho social na carreira apresenta índices de adequação não aceitáveis $\left(X^{2} /\right.$ $g l=8,431$, CFI $=0,787$, GFI $=0,735$, RMSEA $=0,117$, $P[\mathrm{rmsea} \leq 0,05]<0,001)$. Na sequência desta análise, e por terem sido detetados casos extremos multivariados, identificados através da distância quadrada de Mahalanobis, foram desenvolvidos um modelo com correlação entre os fatores de desempenho pobre e excelente (modelo 2.1), um modelo com correlação entre todos os fatores (modelo 2.2) e um modelo com correlação entre todos os fatores sem outliers (modelo 2.3). Este último modelo é o que apresenta melhores resultados $\left(X^{2} / g l=4,097, \mathrm{CFI}=0,895\right.$, GFI $=0,851$, RMSEA $=0,079, P[$ rmsea $\leq 0,05]<0,001)$, com índices de adequação satisfatórios. Os coeficientes de regressão estimados para este modelo são elevados para todos os componentes, e possuem significado estatístico $(p<0,001)$. Os coeficientes das variâncias dos erros e das variáveis latentes também são moderados e significativos do ponto de vista estatístico $(p<0,001)$.

Tabela 2

Índices de Ajustamento Global dos Modelos Geral e de Desempenho Social na Carreira, e Respetivos Modelos Alternativos

\begin{tabular}{|c|c|c|c|c|c|c|c|c|c|}
\hline Modelos & $X^{2}$ & $g l$ & $X^{2} / g l$ & CFI & PCFI & GFI & PGFI & RMSEA & $\begin{array}{c}P \\
(\mathrm{rmsea} \leq 0,05, \\
p=)\end{array}$ \\
\hline 1 - Modelo geral (6 fatores) & $3679,590^{*}$ & 252 & 14,603 & 0,551 & 0,503 & 0,540 & 0,454 & 0,154 & 0,001 \\
\hline $\begin{array}{l}1.1 \text { - Modelo geral com correlação } \\
\text { entre todos os fatores }\end{array}$ & $2998,753^{*}$ & 237 & 12,653 & 0,638 & 0,548 & 0,588 & 0,464 & 0,143 & 0,001 \\
\hline $\begin{array}{l}1.2 \text { - Modelo geral com correlação } \\
\text { entre todos os fatores e sem outliers }\end{array}$ & $2112,735^{*}$ & 237 & 8,914 & 0,716 & 0,615 & 0,649 & 0,512 & 0,127 & 0,001 \\
\hline $\begin{array}{l}2 \text { - Modelo de desempenho social } \\
\text { na carreira ( } 8 \text { fatores) }\end{array}$ & $1995,731^{*}$ & 240 & 8,316 & 0,770 & 0,669 & 0,748 & 0,598 & 0,113 & 0,001 \\
\hline $\begin{array}{l}2.1 \text { - Modelo de desempenho social } \\
\text { na carreira com correlações entre o } \\
\text { desempenho pobre e excelente }\end{array}$ & $1879,844 *$ & 239 & 7,865 & 0,785 & 0,680 & 0,747 & 0,595 & 0,110 & 0,001 \\
\hline $\begin{array}{l}2.2 \text { - Modelo de desempenho social } \\
\text { na carreira com correlação entre todos } \\
\text { os fatores ( } 6 \text { fatores situacionais }+2 \\
\text { fatores de desempenho) }\end{array}$ & $1267,577^{*}$ & 224 & 5,659 & 0,863 & 0,701 & 0,829 & 0,619 & 0,090 & 0,001 \\
\hline $\begin{array}{l}2.3 \text { - Modelo de desempenho social } \\
\text { na carreira com correlações entre } \\
\text { todos os fatores e sem outliers }\end{array}$ & $917,708^{*}$ & 224 & 4,097 & 0,895 & 0,726 & 0,851 & 0,636 & 0,079 & 0,001 \\
\hline
\end{tabular}

$* p<0,001$. 
Pinto, J. C., Taveira, M. C., Candeias, A. \& Araújo, A. (2013). Análise Fatorial Confirmatória da Prova de Avaliação de Competência Social Face à Carreira.

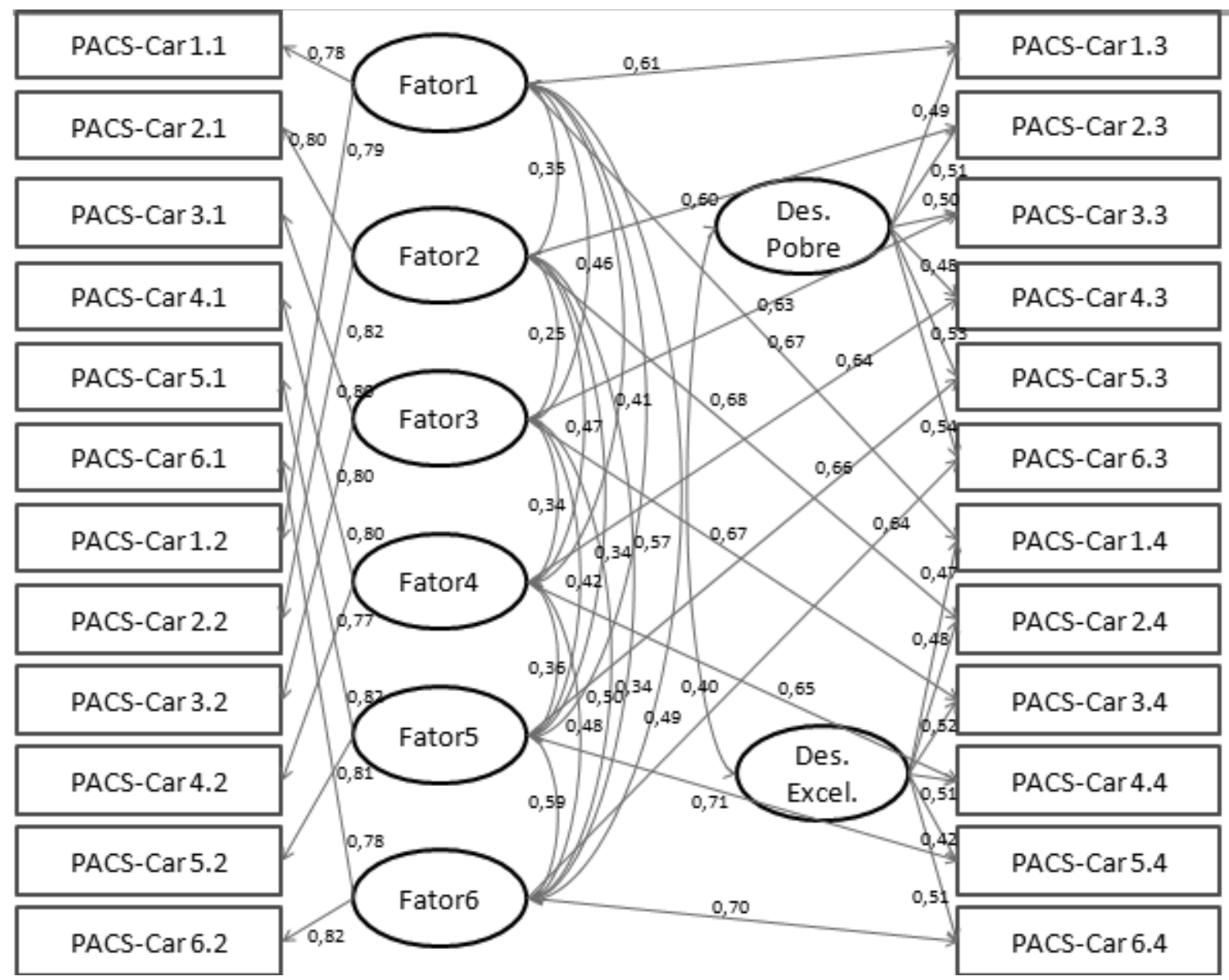

Figura 1. Modelo de desempenho social na carreira com correlações entre todos os fatores e sem outliers.

A Figura 1 apresenta os valores dos pesos fatoriais estandardizados e a fiabilidade individual de cada item do modelo 2.5 .

\section{Discussão e Conclusão}

Ao longo do presente artigo foram apresentados os principais resultados referentes às análises fatoriais, de carácter exploratório e confirmatório, da Prova de Avaliação da Competência Social face à Carreira (PASC-Car). Para o efeito, a prova foi administrada a uma ampla amostra de jovens adolescentes portugueses que se encontravam a frequentar o $8^{\circ}$ ano de escolaridade, em escolas das regiões norte, centro, e sul de Portugal. Os resultados das análises descritivas efetuadas apontam para a existência de alguns problemas relativos à normalidade desta prova. Estes problemas parecem ser uma consequência, por um lado, da formulação e conteúdo dos itens que constituem a prova (Almeida \& Freire, 2003). Ou seja, os itens recorrem à utilização da dupla negação e a sua resposta apela a um pensamento dicotómico, quando questionam o jovem, por exemplo, sobre o grau de dificuldade de determinada situação social e, logo de seguida, sobre o grau de facilidade dessa mesma situação. Uma das formas de tentar ultrapassar os problemas registados quanto ao tipo de resposta solicitada aos participantes seria, por exemplo, a transformação dos atuais itens em itens diferenciais semânticos. Estes consistem em itens bipolares, (e.g. fácil/ difícil; desempenho pobre/desempenho excelente), que integram uma escala de tipo Likert entre os dois adjetivos opostos. A tarefa do participante consiste em determinar primeiramente o adjetivo que melhor o caracteriza, e de seguida, selecionar o ponto na escala que melhor descreve o seu ponto de vista. Por outro lado, estes problemas parecem ser também uma consequência do carácter de novidade associado às situações sociais de carreira que constam na prova, juntamente com o desejo destes jovens transmitirem uma imagem positiva sobre si próprios (por exemplo, indicando que o seu desempenho numa determina situação social fácil "nunca" seria pobre). Neste sentido, estes fatores devem ser tidos em consideração, futuramente, aquando da interpretação dos resultados dos participantes à PACS-Car, para que não sejam colocadas em causa as inferências que deles possam advir (Almeida $\&$ Freire, 2003).

No que concerne à análise fatorial exploratória, os resultados apontam para uma solução fatorial constituída por oito fatores. Apesar de esta conclusão não ser comple- 
tamente congruente com a organização fatorial da prova original (Candeias, 2001, 2008), constituída por seis fatores de natureza situacional, ela apresenta um maior poder explicativo da variância dos resultados. Assim, constata-se a existência de seis fatores relativos a cada uma das situações sociais, e que associam o desempenho ao grau de facilidade ou dificuldade percebida da situação. Constata-se ainda a existência de dois fatores referentes ao desempenho (pobre e excelente), constituídos por itens pertencentes a cada uma das seis situações sociais de carreira. Importa salientar que o estudo de análise fatorial permite analisar o processo a partir do qual as pessoas constroem uma representação mental acerca de um determinado conceito. Neste caso em particular, em virtude da adaptação das situações apresentadas para o contexto de desenvolvimento de carreira, a solução fatorial encontrada na PACS-Car é distinta da solução fatorial do modelo da PACS original. De acordo com estes resultados, parece plausível inferir que o construto de competência social, à semelhança do que acontece já com outros construtos psicológicos (e.g., autoconceito; Marsch \& Shavelson, 1985), não deverá ser sujeito a uma avaliação generalista. A sua estrutura será possivelmente multifacetada, devendo ser percebido como situacionalmente específico, isto é, sensível ao domínio e contexto de aprendizagem social (e.g., Bost, Vaughn, Washington, Cielinski, \& Bradbard, 1998). Por outras palavras, em situações de educação para a carreira, a competência social é percebida de forma diferenciada face a outras situações mais genéricas.

Finalmente, e no que respeita à análise fatorial confirmatória, os resultados obtidos apontam para uma estrutura multidimensional e dinâmica da competência percebida no âmbito da careira, corroborando a complexidade teórica defendida previamente por outros autores (e.g., Candeias \& Almeida, 2005). Por conseguinte, foram testados dois modelos distintos, por comparação com diversos modelos alternativos. $\mathrm{O}$ ajustamento do modelo alternativo de desempenho social na carreira aos dados empíricos é bastante satisfatório. Os resultados indicam que este construto possui uma estrutura marcada pela antecipação de um desempenho pobre ou excelente, transversal a qualquer situação social ou interpessoal. Para além disso, estes resultados mostram que este construto possui ainda uma estrutura definida pela auto perceção de desempenho, em função da conceção de facilidade ou dificuldade, associada a tarefas sociais/interpessoais específicas. Resultados como estes abrem novas pistas para futuras investigações acerca de como estes jovens percebem e diferenciam uma variedade de situações sociais face à carreira.

Perante estes resultados conclui-se que a PACS-Car é uma prova que avalia o desempenho antecipado numa determinada situação social no âmbito da carreira, como consequência da seleção e implementação dos recursos e estratégias cognitivo-comportamentais que possui. A forma como o indivíduo perceciona o seu desempenho, pobre ou excelente, relaciona-se com fatores, tais como, os níveis de autoestima, as crenças de autoeficácia, o estabelecimento de metas e objetivos realistas, a capacidade de iniciativa, a experiência prévia face a situações análogas, e a diversidade, riqueza, e criatividade das estratégias para lidar com desafios sociais (e.g., Candeias, 2008; Cecconello \& Koller, 2000; Del Prette \& Del Prette, 1999; Ford, 1995; Zigler \& Trickett, 1978). Estas variáveis devem ser exploradas de forma mais sistemática com a finalidade de se obter uma perspetiva mais completa sobre a auto perceção de competência social face à carreira em adolescentes. Por outro lado, a PACS-Car é uma prova que tem ainda em consideração a avaliação do grau de facilidade ou dificuldade que o indivíduo atribui a cada situação social no âmbito da carreira. Aqui, as situações sociais apresentadas ganham relevância dado que servem de enquadramento à avaliação que o sujeito faz do seu próprio desempenho neste domínio do desenvolvimento.

\section{Implicações para o Desenvolvimento de Investigações e Intervenções no Ambito da Carreira}

Os resultados obtidos no presente estudo permitem delinear um conjunto de indicações para o aprofundamento teórico e prático desta linha de pesquisa. Em primeiro lugar, sugere-se o aprofundamento do estudo das características psicométricas da PACS-Car, nomeadamente através da replicação deste estudo com uma nova amostra nacional, tendo em vista o esclarecimento dos atuais problemas registados quanto à normalidade da distribuição das respostas à prova (Almeida \& Freire, 2003), e o teste a modelos teóricos alternativos que esclareçam um ajustamento mais adequado aos dados empíricos, incluindo a análise de fatores de segunda ordem (Marôco, 2010). Por outro lado, a adaptação e a aferição das provas de heteroavaliação da competência social aplicada ao contexto de carreira e o estudo das relações deste construto com variáveis vocacionais específicas, como o comportamento exploratório e a capacidade de tomada de decisão, poderão ser vias úteis para uma compreensão integrada do construto da competência social no domínio da carreira.

Relativamente ao desenvolvimento de futuras intervenções, considera-se que os serviços de carreira podem fomentar nos seus clientes o desenvolvimento de comportamentos socialmente inteligentes e competentes neste domínio (Pinto et al., 2011; Taveira et al., 2010). Assim, seria importante que a intervenção vocacional integrasse nos seus objetivos um conjunto de atividades que estimulam os jovens a desenvolver um pensamento e uma compreensão profundas sobre si, qual a sua situação atual de carreira, e qual a situação desejável em termos futuros, transmitindo um sentido de continuidade entre passado, presente e futuro. É também crucial que a intervenção vocacional passe a contemplar atividades que favoreçam nos jovens a oportunidade de adquirir, desenvolver, e implementar, do seu repertório cognitivo-comportamental, as atitudes e comportamentos mais adequados à situação atual, aumentando as possibilidades de realização de objetivos pessoais, académicos, profissionais e sociais. 
Pinto, J. C., Taveira, M. C., Candeias, A. \& Araújo, A. (2013). Análise Fatorial Confirmatória da Prova de Avaliação de Competência Social Face à Carreira.

Finalmente, é ainda fundamental que a intervenção vocacional ajude, desde cedo, os jovens a conciliarem a observação das suas próprias características, objetivos e planos para o futuro com uma exploração e reflexão profunda em torno das características e necessidades dos seus contextos interpessoais e sociais, bem como, das mútuas influências entre si e estes contextos. Os projetos de vida desenvolvidos pelos jovens devem ser contextualizados, e ter em consideração um questionamento ético que assegure o respeito pelos direitos humanos socialmente instituídos. O questionamento ético no âmbito da carreira envolve a capacidade para refletir de modo probabilístico, e a partir de múltiplas perspetivas, sobre as implicações dos seus ideais e opções de carreira e de vida. Envolve a capacidade para se questionar acerca das implicações das suas decisões e estilo devida desejado, quer para si próprio/a (em termos de benefícios individuais, como satisfação e sucesso), quer para os outros mais próximos e significativos (pais, amigos, companheiros), e quer ainda, para o Outro mais distante (a sociedade, em termos de justiça social e sustentabilidade). Esta capacidade e envolvimento na reflexividade ética na carreira requerem níveis de pensamento formal e de competências de desenvolvimento moral pós-convencional, em geral observadas nos anos mais tardios da adolescência. No entanto, estas podem ser estimuladas antes, a par da competência social, que incide sobre informações relativas e ao próprio sujeito, aos outros indivíduos (suas emoções e interesses, seus pensamentos e comportamentos), e às interações entre eles.

\section{Referências}

Alexander, K. L., Entwisle, D. R., \& Dauber, S. L. (1993). First-grade classroom behavior: Its short and long-term consequences for school performance. Child Development, 64, 801-814.

Almeida, L. S., \& Freire, T. (2003). Metodologia da investigação em Psicologia e Educação (3. ed). Braga, Portugal: Psiquilíbrios.

Araújo, A., Taveira, M. C., \& Candeias, A. A. (2009). Prova de Avaliação de Competência Social em Contexto de Carreira (PACS-Car). Braga, Portugal: Universidade do Minho.

Bandeira, M., Del Prette, Z. A. P., Del Prette, A., \& Magalhães, T. (2009). Escala de avaliação de habilidades sociais de estudantes do ensino fundamental, SSRS-BR: Validação transcultural para o Brasil. Psicologia: Teoria e Pesquisa, 25(2), 271-282.

Bandura, A. (1986). Social foundations of thought and action: A social cognitive theory. Englewood Cliffs, NJ: Prentice-Hall.

Bandura, A., Barbaranelli, C., Caprara, G. V., \& Pastorelli, C. (2001). Self-efficacy beliefs as shapers of children's aspirations and career trajectories. Child Development, 72(1), 187-206.

Barnes, M., \& Sternberg, R. J. (1989). Social intelligence and decoding of nonverbal cues. Intelligence, 13, 263-287.

Bost, K. K., Vaughn, B. E., Washington, W. N., Cielinski, K. L., \& Bradbard, M. R. (1998). Social competence, social support, and attachment: Demarcation of construct domains, measurement, and paths of influence for preschool children attending Head Start. Child Development, 69, 192-218.
Bruder, M. B., \& Chen, L. H. (2007). Measuring social competence in toddlers: Play tools for learning. Early Childhood Services: An Interdisciplinary Journal of Effectiveness, 1(1), 49-70.

Candeias, A. A. (2001). Prova de Avaliação de Competência Social. Évora, Portugal: Universidade de Évora.

Candeias, A. A. (2008). Prova de Avaliação de Competência Social. Évora, Portugal: Universidade de Évora.

Candeias, A. A., \& Almeida, L. S. (2005). Competência social: A sua avaliação em contextos de desenvolvimento e educação. Revista de Psicologia, Educação e Cultura, 2(9), 359-378.

Carneiro, R. S., Falcone, E., Clark, C., Del Prette, Z., \& Del Prette, A. (2007). Qualidade de vida, apoio social e depressão em idosos: Relação com habilidades sociais. Psicologia: Reflexão e Crítica, 20(2), 229-237.

Carvalho, V. (2011). Inteligência Social em Alunos do $8^{\circ}$ ano: Implicações para o Questionamento Ético dos Projectos de Vida (Dissertação de mestrado, Escola de Psicologia, Universidade do Minho, Braga, Portugal).

Cecconello, A. M., \& Koller, S. H. (2000). Competência social e empatia: Um estudo sobre resiliência com crianças em situação de pobreza. Estudos de Psicologia (Natal), 5(1), 71-93.

Cotugno, A. J. (2009). Social competence and social skills training and intervention with autism spectrum disorders. Journal of Autism and Developmental Disorders, 39(9), 1268-1277.

Del Prette, Z. A. P., \& Del Prette, A. (1999). Psiologia das habilidades sociais (4. ed.). Petrópolis, RJ: Vozes.

Del Prette, Z. A. P., \& Del Prette, A. (2005). Psicologia das habilidades sociais na infância: teoria e prática (2. ed.). Petrópolis. RJ: Vozes.

Duarte, M. E., Lassance, M. C., Savickas, M. L., Rossier, J. Dauwalder, J. P., Guichard, ...Vianen, A. E. M. (2009). A construção da vida: Um novo paradigma para entender a carreira no século XXI. Revista Interamericana de Psicologia, 44(2), 392-406.

Feitosa, F. B. (2007). Habilidades sociais e desempenho académico: Processos cognitivos como moderadores e mediadores (Dissertação de doutorado, Centro de Educação e Ciências Humanas, Universidade Federal de São Carlos, São Carlos, SP, Brasil).

Ford, M. (1982). Social cognition and social competence in adolescence. Developmental Psychology, 18, 323-340.

Ford, M. (1986). For all practical purposes: Criteria for defining and evaluating practical intelligence. In R. Sternberg \& R. Wagner (Eds.), Practical intelligence: Nature and origins of competence in the everyday competence (pp. 183-201): Cambridge, UK: Cambridge University Press.

Ford, M. (1995). Intelligence and personality in social behavior In D. Saklofske \& M. Zeidner (Eds.), International handbook of personality and intelligence (pp. 125-140). New York: Plenum Press.

Fagoulis, I., \& Phillips, N. (2011). Social skills for successful career development. Review of European Studies, 3(1), 85-93.

Fumo, V. M. S. (2009). Habilidades sociais académicas de crianças com baixo e alto desempenho académico na interação com o professor (Dissertação de mestrado, Centro de Educação e Ciências Humanas, Universidade Federal de São Carlos, São Carlos, SP, Brasil).

Gouley, K. K., Brotman, L. M., Huang, K. Y., \& Shrout, P. E. (2008). Construct validation of the Social Competence Scale in Preschool-age children. Social Development, 17(2), 380-398. 
Greenspan, S., \& Driscoll, J. (1997). The role of intelligence in a broad model of personal competence. In D. Flanagan, J. Genshaft, \& P. Harrison (Eds.), Contemporary intellectual assessment: Theories, tests, and issues (pp. 131-150). London: The Guilford Press.

Gresham, F. M., \& Elliot, S. (1984). Assessment and classification of children's social skills: A review of methods and issues. School Psychology Review, 13, 292-301.

Gresham, F., \& Elliot, S. (1990). Social skills rating system. Circle Pines, MN: American Guidance Service.

Gresham, F. M., Sugai, G., \& Horner, R. H. (2001). Interpreting outcomes of social skills training in students with highincidence disabilities. The Council for Exceptional Children, 67(3), 331-344.

Guichard, J. (2011). How to develop their careers and design their lives in Western Societies at the beginning of the $21^{\text {st }}$ century. Trabalho apresentado na Faculdade de Psicologia e Ciências da Educação, Universidade de Lisboa, Portugal.

Guichard, J., \& Huteau, M. (2001). Psychologie de l'orientation. Paris: Dunod.

Henricsson, L., \& Rydel, A. M. (2006). Children with behavior problems: The influence of social competence and social relations on problem stability, school achievement and peer acceptance across the first six years of school. Infant and Child Development, 15, 347-366.

Hoyt, K. B., \& Shylo, K. R. (1987). Career education in transition: Trends and implications for the future. Columbus: ERIC Clearinghouse on Adult, Career, and Vocational Education, The Ohio State University.

Ladd, G. W. (1999). Peer relationships and social competence during early and middle childhood. Annual Reviews in Psychology, 50, 333-359.

Lemos, M. S., \& Meneses, H. I. (2002). A avaliação da competência social: Versão portuguesa da forma para professores do SSRS. Psicologia: Teoria e Pesquisa, 18(3), 267-274.

Malecki, C. K., \& Elliot, S. N. (2002). Children's social behavior as predictors of academic achievement: A longitudinal analysis. School Psychology Quarterly, 17, 1-23.

Marôco, J. (2010). Análise de Equações Estruturais. Lisboa, Portugal: ReportNumber.

Marsch, H. W., \& Shavelson, R. (1985). Self-concept: Its multifaceted hierarquical structure. Educational Psychologist, 20, 107-123.

Molina, R. C. M., \& Dell Prette, Z. A. P. (2006). Funcionalidade da relação entre habilidades sociais e dificuldade de aprendizagem. Psico-UFS, 11(1), 53-63.

Murakami, K., Murray, L., Sims, D., \& Chedzey, K. (2009). Learning on work placement: The narrative development of social competence. Journal of Adult Development, 16, 13-24.
Nunnally, J. C. (1978). Psychometric theory. New York: McGraw-Hill.

Pinto, J. C., Taveira, M. C., Candeias, A., \& Araújo, A. (2011, $25-$ 27 jul.). Avaliação da competência social face à carreira em estudantes do $8^{\circ}$ ano de escolaridade. Trabalho apresentado no VII Congresso Ibero-americano de Avaliação Psicológica, Lisboa, Portugal.

Salmela-Aro, K., Tolvanen, A., \& Nurmi, J. E. (2011). Social strategies during university studies predict early career work burnout and engagement: 18-year longitudinal study. Journal of Vocational Behavior, 79, 145-157.

Savickas, M. L. (2005). The theory and practice of career construction. In D. Brown \& R. Lent (Eds.), Career development and counselling: Putting theory and research to work (pp. 43-70). New York: John Wiley \& Sons.

Savickas, M. L., Nota, L., Rossier, J., Dauwalder, J. P., Duarte, M. E., Guichard, J., ...Vianen, A. E. M. (2009). Life-designing: A paradigm for career construction in the $21^{\text {st }}$ century. Journal of Vocational Behavior, 75(3), 239-250.

Schoon, I. (2009, March). Measuring social competencies. In German Council for Social and Economic Data, Working Paper Series of the Council for Social and Economic Data (Working paper No. 58). Berlin, Germany: Federal Ministry of Education and Research.

Segrin, C., Hazel, A., Donnerstein, C., Taylor, M., \& Domschke, T. (2007). Social skills, psychological well-being and the mediating role of perceived stress. Anxiety, Stress \& Coping, 20(3), 321-329.

Sternberg, R. J., \& Grigorenko, E. (2003). Evaluación dinámica del potencial de aprendizaje. Barcelona, España: Paidos.

Taveira, M. C., Saavedra, L., Silva, A. D., Araújo, A., Faria, L., Pinto, J. C., ...Pereira, A. (2010). O plano de avaliação do projecto EQ4CCi. Carreira e cidadania: Condições pessoais e do contexto para o questionamento ético dos projectos de vida. In M. C. Taveira \& A. D. Silva (Coords.), Desenvolvimento Vocacional: Avaliação e Intervenção (pp. 217-224). Braga, Portugal: Associação Portuguesa para o Desenvolvimento da Carreira.

Tyler, F. (1984). El comportamiento psicosocial, la competencia psicosocial individual y las redes de intercambio de recursos como ejemplos de psicología comunitária. Revista Latino-Americana de Psicologia, 16, 77-92.

Welsh, M., Parke, R. D., Widaman, K., \& O'Neil, R. (2001). Linkages between children's social and academic competence: A longitudinal analysis. Journal of School Psychology, 39(6), 463-481.

Zigler, E., \& Trickett, P. K. (1978). IQ, social competence, and evaluation of early childhood intervention programs. American Psychologist, 33, 789-798.

\section{Errata:}

No artigo "Análise Fatorial Confirmatória da Prova de Avaliação de Competência Social Face à Carreira”, 26(3)2013, p. 469, onde se lia: Joana Carneiro Pinto, Maria do Céu Taveira, Adelinda Candeias (Universidade Católica Portuguesa, Lisboa, Distrito de Lisboa, Portugal) \& Alexandra Araújo (Universidade de Évora, Évora, Distrito de Évora, Portugal)

Leia-se: Joana Carneiro Pinto (Universidade Católica Portuguesa, Lisboa, Distrito de Lisboa, Portugal), Maria do Céu Taveira (Universidade do Minho, Minho, Distrito do Minho, Portugal), Adelinda Candeias (Universidade de Évora, Évora, Distrito de Évora, Portugal) \& Alexandra Araújo (Universidade do Minho, Minho, Distrito do Minho, Portugal). 